\title{
Crystal Structure of Dimeric [4-\{(2-Oxybenzyl)imino\}-2-penten-2-olato- $\left.O, O^{\prime}, N\right] \operatorname{copper}($ II) Complex
}

\author{
Dinçer ÜlLKü*, Cengiz ArICI*, M. Nawaz TAHIR*, Orhan ATAKoL ${ }^{* * \dagger}$ and Mustafa TAŞTEKIN** \\ *Department of Engineering Physics, Hacettepe University, Beytepe 06532, Ankara, Turkey \\ **Department of Chemistry, Science Faculty, Ankara University, Tandogan 06100, Turkey
}

Due to the super exchange mechanism over the $\mathrm{O}$ atoms, dimeric copper(II) complexes with double oxygen bridges show subnormal magnetic moments. ${ }^{1-3}$ There seems to be correlations between the magnetic properties of the systems and the dihedral angle between the bridging plane and the coordination square around copper, as well as with the $\mathrm{Cu}-\mathrm{O}-\mathrm{Cu}$ bridging angle. As a contribution of our previous work $^{4-7}$ on dimeric $\mathrm{Cu}$ complexes, this structure determination was performed to obtain more information about the stereochemistry around the copper atom.

The crystalline ligand 4-[(2-hydroxybenzyl)imino]-2hydroxy-2-penten was prepared from the mixture of 2hydroxybenzylamine $(1.23 \mathrm{~g} ; 0.01 \mathrm{~mol})$ and acetylacetone $(1.0 \mathrm{~g} ; 0.01 \mathrm{~mol})$ in $30 \mathrm{ml}$ of hot ethanol after $24 \mathrm{~h}$ in air. To a solution of the ligand $(0.205 \mathrm{~g} ; 1 \mathrm{mmol})$ in $40 \mathrm{ml}$ DMF above $130^{\circ} \mathrm{C}$, a solution of $\mathrm{Cu}\left(\mathrm{CH}_{3} \mathrm{COO}\right)_{2}$. $\mathrm{H}_{2} \mathrm{O}(0.199 \mathrm{~g} ; 1 \mathrm{mmol})$ in $20 \mathrm{ml}$ hot methanol was added dropwise and the mixture was set aside. The crystals were obtained after allowing the solution to stand for $36 \mathrm{~h}$ at room temperature. The chemical reaction of the title compound is shown in Fig. 1.

The complex includes two $\mathrm{Cu}\left(\mathrm{C}_{12} \mathrm{H}_{13} \mathrm{NO}_{2}\right)$ structure units linked via phenolic $\mathrm{O}$ atoms (Fig. 2). The central $\mathrm{CuOCuO}$ bridging group is practically planar. The $\mathrm{Cu}$ and $\mathrm{O}$ atoms lie alternating $0.009 \AA$ above and below this least squares plane. The $\mathrm{Cu} 1-\mathrm{N} 1$ and $\mathrm{Cu} 2-\mathrm{N} 2$ bond lengths are 1.906(4) and 1.925(4) $\mathrm{A}$, respectively. The average $\mathrm{Cu}-\mathrm{O} 1 \quad[1.871(2) \AA]$ and $\mathrm{Cu}-\mathrm{O} 2$ [1.946(9) $\AA]$ bond distances could be considered equal within experimental error. The average value of the $\mathrm{Cu}-\mathrm{O}-\mathrm{Cu}$ bridging angle is $102.8(3)^{\circ}$. These values are

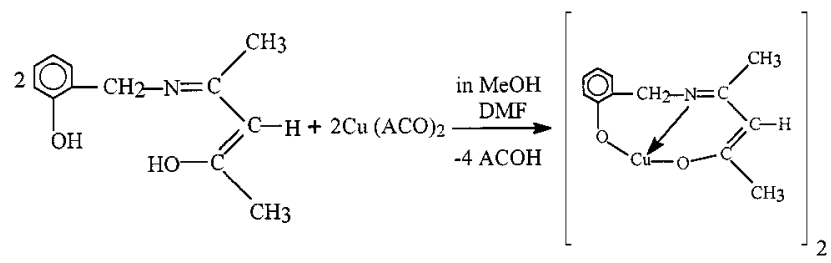

Fig. 1 Synthesis and chemical structure.

\footnotetext{
$\doteqdot$ To whom correspondence should be addressed.
}

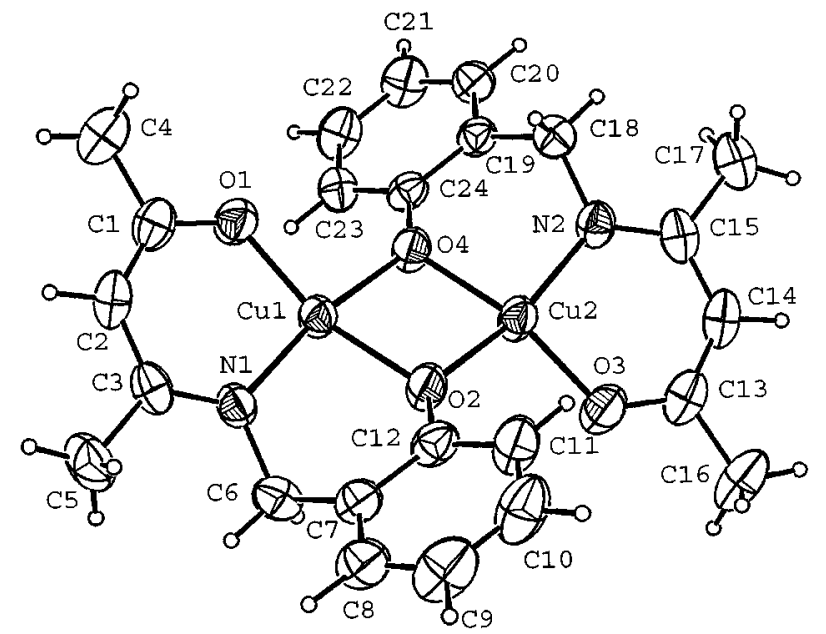

Fig. 2 The ORTEP drawing of the titled compound with atom labeling.

Table 1 Crystal and experimental data

Formula: $\mathrm{C}_{24} \mathrm{H}_{26} \mathrm{Cu}_{2} \mathrm{~N}_{2} \mathrm{O}_{4}$

Formula weight $=533.57$

Crystal system: orthorhombic

Space group: $P \mathrm{ca} 2{ }_{1} \quad Z=4$

$a=21.885(3) \AA$

$b=12.972(3) \AA$

$c=8.082(2) \AA$

$V=2294.4(8) \AA^{3}$

$D_{\mathrm{x}}=1.544 \mathrm{~g} / \mathrm{cm}^{3}$

$\mu\left(\mathrm{Mo} \mathrm{K}_{\alpha}\right)=1.890 \mathrm{~mm}^{-1}$

$T=295 \mathrm{~K}$

Dark

$F\left(\begin{array}{lll}0 & 0 & 0\end{array}\right)=1096$

Crystal size: $0.5 \times 0.4 \times 0.35 \mathrm{~mm}$

Radiation $=\mathrm{Mo} \mathrm{K}_{\alpha}$

$R=0.029$

$R_{W}=0.035$

No. of reflections used $=2232$

No. of parameters $=294$

Goodness-of-fit $=0.94$

Measurements: Enraf Nonius CAD-4 diffractometer

Program system: CAD-4 EXPRESS Software

Structure determination: MolEN

Treatment of hydrogen atoms: geometric calculation Refinement: full matrix least-squares (MolEN) 
Table 2 Final atomic coordinates and equivalent isotropic thermal parameters for non-hydrogen atoms

\begin{tabular}{cllll}
\hline Atom & \multicolumn{1}{c}{$x$} & \multicolumn{1}{c}{$y$} & \multicolumn{1}{c}{$z$} & $B_{\mathrm{eq}} / \AA^{2}$ \\
\hline $\mathrm{C} 11$ & $0.43842(2)$ & $0.27842(3)$ & 0.110 & $3.061(8)$ \\
$\mathrm{C} 2$ & $0.31676(2)$ & $0.18031(4)$ & $0.2043(1)$ & $3.494(9)$ \\
$\mathrm{O} 1$ & $0.5185(1)$ & $0.2338(2)$ & $0.1486(5)$ & $3.90(7)$ \\
$\mathrm{O} 2$ & $0.3529(1)$ & $0.3136(2)$ & $0.1484(5)$ & $3.86(7)$ \\
$\mathrm{O} 3$ & $0.2358(1)$ & $0.2226(2)$ & $0.1759(5)$ & $4.48(8)$ \\
$\mathrm{O} 4$ & $0.4021(1)$ & $0.1461(2)$ & $0.1704(4)$ & $3.40(6)$ \\
$\mathrm{N} 1$ & $0.4588(2)$ & $0.4114(3)$ & $0.0262(5)$ & $3.16(7)$ \\
$\mathrm{N} 2$ & $0.2991(2)$ & $0.0558(3)$ & $0.3249(5)$ & $3.27(7)$ \\
$\mathrm{C} 1$ & $0.5660(2)$ & $0.2922(3)$ & $0.1342(6)$ & $3.61(9)$ \\
$\mathrm{C} 2$ & $0.5656(2)$ & $0.3915(4)$ & $0.0780(7)$ & $4.0(1)$ \\
$\mathrm{C} 3$ & $0.5142(2)$ & $0.4495(3)$ & $0.0259(6)$ & $3.41(8)$ \\
$\mathrm{C} 4$ & $0.6247(2)$ & $0.2445(4)$ & $0.1918(9)$ & $5.2(1)$ \\
$\mathrm{C} 5$ & $0.5270(2)$ & $0.5604(4)$ & $-0.0238(8)$ & $5.0(1)$ \\
$\mathrm{C} 6$ & $0.4055(2)$ & $0.4735(4)$ & $-0.0265(6)$ & $3.9(1)$ \\
$\mathrm{C} 7$ & $0.3625(2)$ & $0.4938(3)$ & $0.1127(7)$ & $3.41(8)$ \\
$\mathrm{C} 8$ & $0.3466(2)$ & $0.5933(3)$ & $0.1603(7)$ & $4.2(1)$ \\
$\mathrm{C} 9$ & $0.3051(2)$ & $0.6106(4)$ & $0.2851(9)$ & $5.5(1)$ \\
$\mathrm{C} 10$ & $0.2794(2)$ & $0.5277(4)$ & $0.3651(9)$ & $5.5(1)$ \\
$\mathrm{C} 11$ & $0.2952(2)$ & $0.4279(4)$ & $0.3254(8)$ & $4.5(1)$ \\
$\mathrm{C} 12$ & $0.3358(2)$ & $0.4103(3)$ & $0.1948(7)$ & $3.44(8)$ \\
$\mathrm{C} 13$ & $0.1891(2)$ & $0.1713(4)$ & $0.2272(7)$ & $3.90(9)$ \\
$\mathrm{C} 14$ & $0.1910(2)$ & $0.0792(4)$ & $0.3090(7)$ & $3.90(9)$ \\
$\mathrm{C} 15$ & $0.2438(2)$ & $0.0231(3)$ & $0.3572(6)$ & $3.35(8)$ \\
$\mathrm{C} 16$ & $0.1284(2)$ & $0.2201(4)$ & $0.188(1)$ & $5.4(1)$ \\
$\mathrm{C} 17$ & $0.2326(2)$ & $-0.0776(4)$ & $0.4473(7)$ & $4.6(1)$ \\
$\mathrm{C} 18$ & $0.3536(2)$ & $-0.0007(4)$ & $0.3832(7)$ & $3.63(9)$ \\
$\mathrm{C} 19$ & $0.3962(2)$ & $-0.0299(3)$ & $0.2448(6)$ & $2.93(8)$ \\
$\mathrm{C} 20$ & $0.4137(2)$ & $-0.1307(3)$ & $0.2190(7)$ & $3.61(9)$ \\
$\mathrm{C} 21$ & $0.4546(2)$ & $-0.1560(3)$ & $0.0944(8)$ & $4.4(1)$ \\
$\mathrm{C} 22$ & $0.4779(2)$ & $-0.0804(4)$ & $-0.0033(7)$ & $4.2(1)$ \\
$\mathrm{C} 23$ & $0.4613(2)$ & $0.0221(4)$ & $0.0194(7)$ & $3.71(9)$ \\
$\mathrm{C} 24$ & $0.4203(2)$ & $0.0469(3)$ & $0.1447(5)$ & $2.85(8)$ \\
& & & &
\end{tabular}

$B_{\mathrm{eq}}=\left(8 \pi^{2} / 3\right) \sum_{i} \sum_{j} U_{i j} a_{i}^{*} a_{j}^{*}\left(\boldsymbol{a}_{i} \cdot \boldsymbol{a}_{j}\right)$.

comparable with the corresponding values reported in the literature..$^{4-7}$ The displacement of the $\mathrm{Cu} 1$ and $\mathrm{Cu} 2$ atoms from their coordination best planes are 0.0861(1) and $0.0378(9) \AA$ respectively. The dihedral angles between the bridging plane and the coordination planes around $\mathrm{Cu} 1$ and $\mathrm{Cu} 2$ are $9.9(3)^{\circ}$ and $12.6(3)^{\circ}$ respectively. The $\mathrm{Cu}-\mathrm{Cu}$ separation $[3.0478(6) \AA]$ is comparable with the values found in the reported structures. ${ }^{4-7}$ Table 1 shows the crystal and experimental data, while final atomic parameters are given in Table 2. The bond distances and angles are shown in Table 3.

\section{References}

1. R. J. Butcher and E. Sinn, Inorg. Chem., 15, 1604 (1976).

2. M. Kato, H. B. Jonassen and J. C. Fanning, Chem. Rev., 99 (1964).

3. M. Kato and Y. Muto, Coord. Chem. Rev., 45 (1988).

4. M. N. Tahir, D. Ülkü, O. Atakol and A. Akay, Acta Crystallogr., C52, 2676 (1996).

5. D. Ülkü, F. Ercan, O. Atakol, I. Ercan and A. Gencer, Acta
Table 3 Bond distances $(\AA)$ and angles $\left({ }^{\circ}\right)$

\begin{tabular}{|c|c|c|c|}
\hline $\mathrm{Cu} 1-\mathrm{O} 1$ & $1.872(3)$ & $\mathrm{C} 3-\mathrm{C} 5$ & $1.520(7)$ \\
\hline $\mathrm{Cu} 1-\mathrm{O} 2$ & $1.952(3)$ & $\mathrm{C} 6-\mathrm{C} 7$ & $1.490(8)$ \\
\hline $\mathrm{Cu} 1-\mathrm{O} 4$ & $1.954(3)$ & $\mathrm{C} 7-\mathrm{C} 8$ & $1.391(7)$ \\
\hline $\mathrm{Cu} 1$ - N1 & $1.906(4)$ & C7 - C12 & $1.397(7)$ \\
\hline $\mathrm{Cu}_{2}-\mathrm{O}_{2}$ & $1.954(3)$ & $\mathrm{C} 8-\mathrm{C} 9$ & $1.376(9)$ \\
\hline $\mathrm{Cu} 2-\mathrm{O} 3$ & $1.869(3)$ & C9 - C10 & $1.374(9)$ \\
\hline $\mathrm{Cu} 2-\mathrm{O} 4$ & $1.939(3)$ & C10 - C11 & $1.378(8)$ \\
\hline $\mathrm{Cu} 2-\mathrm{N}_{2}$ & $1.925(4)$ & $\mathrm{C} 11-\mathrm{C} 12$ & $1.399(8)$ \\
\hline $\mathrm{O} 1-\mathrm{C} 1$ & $1.291(5)$ & $\mathrm{C} 13-\mathrm{C} 14$ & $1.366(8)$ \\
\hline $\mathrm{O} 2-\mathrm{C} 12$ & $1.361(5)$ & $\mathrm{C} 13-\mathrm{C} 16$ & $1.504(7)$ \\
\hline $\mathrm{O} 3-\mathrm{C} 13$ & $1.289(6)$ & C14 - C15 & $1.420(8)$ \\
\hline $\mathrm{O} 4 \cdot \mathrm{C} 24$ & $1.363(5)$ & $\mathrm{C} 15-\mathrm{C} 17$ & $1.516(7)$ \\
\hline $\mathrm{N} 1-\mathrm{C} 3$ & $1.309(6)$ & C18 - C19 & $1.505(7)$ \\
\hline N1 - C6 & $1.481(6)$ & $\mathrm{C} 19-\mathrm{C} 20$ & $1.378(6)$ \\
\hline $\mathrm{N} 2-\mathrm{C} 15$ & $1.309(7)$ & C19- C24 & $1.387(6)$ \\
\hline $\mathrm{N} 2-\mathrm{C} 18$ & $1.476(6)$ & $\mathrm{C} 20-\mathrm{C} 21$ & $1.387(8)$ \\
\hline $\mathrm{C} 1-\mathrm{C} 2$ & $1.366(7)$ & $\mathrm{C} 21-\mathrm{C} 22$ & $1.358(8)$ \\
\hline $\mathrm{C} 1-\mathrm{C} 4$ & $1.500(7)$ & $\mathrm{C} 22-\mathrm{C} 23$ & $1.390(7)$ \\
\hline $\mathrm{C} 2-\mathrm{C} 3$ & $1.418(7)$ & $\mathrm{C} 23-\mathrm{C} 24$ & $1.390(7)$ \\
\hline $\mathrm{O} 1-\mathrm{Cu} 1-\mathrm{O} 2$ & $160.7(2)$ & $\mathrm{N} 1-\mathrm{C} 3-\mathrm{C} 5$ & $121.9(5)$ \\
\hline $\mathrm{O} 1-\mathrm{Cu} 1-\mathrm{O} 4$ & $93.9(1)$ & $\mathrm{C} 2-\mathrm{C} 3-\mathrm{C} 5$ & $115.8(5)$ \\
\hline $\mathrm{O} 1-\mathrm{Cu} 1-\mathrm{N} 1$ & $96.9(2)$ & $\mathrm{N} 1-\mathrm{C} 6-\mathrm{C} 7$ & $112.1(4)$ \\
\hline $\mathrm{O} 2-\mathrm{Cu} 1-\mathrm{O} 4$ & $77.0(1)$ & $\mathrm{C} 6-\mathrm{C} 7-\mathrm{C} 8$ & $122.0(5)$ \\
\hline $\mathrm{O} 2-\mathrm{Cul}-\mathrm{N} 1$ & $94.0(1)$ & $\mathrm{C} 6-\mathrm{C} 7-\mathrm{C} 12$ & $119.0(4)$ \\
\hline $\mathrm{O} 4-\mathrm{Cu} 1-\mathrm{N} 1$ & $168.3(2)$ & $\mathrm{C} 8-\mathrm{C} 7-\mathrm{C} 12$ & $118.9(5)$ \\
\hline $\mathrm{O} 2-\mathrm{Cu} 2-\mathrm{O} 3$ & $95.5(1)$ & $\mathrm{C} 7-\mathrm{C} 8-\mathrm{C} 9$ & $121.2(5)$ \\
\hline $\mathrm{O} 2-\mathrm{Cu} 2-\mathrm{O} 4$ & $77.3(1)$ & $\mathrm{C} 8-\mathrm{C} 9-\mathrm{C} 10$ & $119.2(5)$ \\
\hline $\mathrm{O} 2-\mathrm{Cu} 2-\mathrm{N} 2$ & $160.1(2)$ & $\mathrm{C} 9-\mathrm{C} 10-\mathrm{C} 11$ & $121.5(6)$ \\
\hline $\mathrm{O} 3-\mathrm{Cu} 2-\mathrm{O} 4$ & $164.4(2)$ & C10 - C11 - C12 & $119.3(6)$ \\
\hline $\mathrm{O} 3-\mathrm{Cu} 2-\mathrm{N} 2$ & $96.8(2)$ & $\mathrm{O} 2-\mathrm{C} 12-\mathrm{C} 7$ & $118.0(5)$ \\
\hline $\mathrm{O} 4-\mathrm{Cu} 2-\mathrm{N} 2$ & $94.1(1)$ & $\mathrm{O} 2-\mathrm{C} 12-\mathrm{C} 11$ & $122.2(5)$ \\
\hline $\mathrm{Cu} 1-\mathrm{O} 1-\mathrm{Cl}$ & $123.9(3)$ & $\mathrm{C} 7-\mathrm{C} 12-\mathrm{C} 11$ & $119.8(4)$ \\
\hline $\mathrm{Cu} 1-\mathrm{O} 2-\mathrm{Cu} 2$ & $102.6(1)$ & $\mathrm{O} 3-\mathrm{C} 13-\mathrm{Cl} 4$ & $125.6(4)$ \\
\hline $\mathrm{Cu} 1-\mathrm{O} 2-\mathrm{C} 12$ & $121.5(3)$ & $\mathrm{O} 3-\mathrm{Cl} 3-\mathrm{C} 16$ & $114.6(5)$ \\
\hline $\mathrm{Cu} 2-\mathrm{O} 2-\mathrm{C} 12$ & $129.9(3)$ & C14 - C13 - C16 & $119.8(5)$ \\
\hline $\mathrm{Cu} 2-\mathrm{O} 3 \cdot \mathrm{C} 13$ & $124.2(3)$ & $\mathrm{C} 13-\mathrm{C} 14-\mathrm{C} 15$ & $127.3(4)$ \\
\hline $\mathrm{Cu} 1-\mathrm{O} 4-\mathrm{Cu} 2$ & $103.0(1)$ & $\mathrm{N} 2-\mathrm{C} 15-\mathrm{C} 14$ & $122.1(4)$ \\
\hline $\mathrm{Cul}-\mathrm{O} 4-\mathrm{C} 24$ & $132.2(3)$ & N2 - C15 - C17 & $121.7(5)$ \\
\hline $\mathrm{Cu} 2-\mathrm{O} 4-\mathrm{C} 24$ & $121.3(2)$ & $\mathrm{C} 14-\mathrm{C} 15-\mathrm{C} 17$ & $116.2(5)$ \\
\hline $\mathrm{Cu} 1$ - N1 - C3 & $124.0(3)$ & N2 - C18 - C19 & $112.8(5)$ \\
\hline $\mathrm{Cu} 1$ - N1 - C6 & $114.2(3)$ & $\mathrm{C} 18-\mathrm{C} 19-\mathrm{C} 20$ & $121.6(4)$ \\
\hline $\mathrm{C} 3$ - N1 - C6 & $121.6(4)$ & $\mathrm{C} 18-\mathrm{C} 19-\mathrm{C} 24$ & $119.2(4)$ \\
\hline $\mathrm{Cu} 2-\mathrm{N} 2-\mathrm{C} 15$ & $123.9(4)$ & $\mathrm{C} 20-\mathrm{C} 19-\mathrm{C} 24$ & $119.2(4)$ \\
\hline $\mathrm{Cu} 2-\mathrm{N} 2-\mathrm{C} 18$ & $114.6(3)$ & $\mathrm{C} 19-\mathrm{C} 20-\mathrm{C} 21$ & $120.9(5)$ \\
\hline $\mathrm{C} 15-\mathrm{N} 2-\mathrm{C} 18$ & $121.4(4)$ & $\mathrm{C} 20-\mathrm{C} 21-\mathrm{C} 22$ & $119.6(4)$ \\
\hline $\mathrm{O} 1-\mathrm{C} 1-\mathrm{C} 2$ & $125.3(4)$ & $\mathrm{C} 21-\mathrm{C} 22-\mathrm{C} 23$ & $121.0(5)$ \\
\hline $\mathrm{O} 1-\mathrm{C} 1-\mathrm{C} 4$ & $114.8(5)$ & $\mathrm{C} 22-\mathrm{C} 23-\mathrm{C} 24$ & $119.1(5)$ \\
\hline $\mathrm{C} 2-\mathrm{C} 1-\mathrm{C} 4$ & $119.8(4)$ & $\mathrm{O} 4-\mathrm{C} 24-\mathrm{C} 19$ & $118.5(4)$ \\
\hline $\mathrm{C} 1-\mathrm{C} 2-\mathrm{C} 3$ & $127.2(4)$ & $\mathrm{O} 4-\mathrm{C} 24-\mathrm{C} 23$ & $121.2(4)$ \\
\hline $\mathrm{N} 1-\mathrm{C} 3-\mathrm{C} 2$ & $122.3(4)$ & C19-C24 - C23 & $120.2(4)$ \\
\hline
\end{tabular}

Crystallogr., C53, 179 (1997).

6. O. Atakol, F. Ercan, D. Ülkü and N. Y1lmaz, Anal. Sci., 13, 1051 (1997).

7. M. N. Tahir, D. Ülkü, M. Taştekin and O. Atakol, Anal. Sci., 13, 1055 (1997).

(Received August 26, 1997) (Accepted November 25, 1997) 